\title{
Jan Plamper, Geschichte und Gefühl. Grundlagen der
} Emotionsgeschichte

München : Siedler, 2012, 478 p., 29,99€.

\section{Falk Bretschneider}

\section{OpenEdition}

\section{Journals}

Édition électronique

URL : http://journals.openedition.org/ifha/8358

DOI : 10.4000/ifha.8358

ISSN : 2198-8943

Éditeur

IFRA - Institut franco-allemand (sciences historiques et sociales)

Référence électronique

Falk Bretschneider, « Jan Plamper, Geschichte und Gefühl. Grundlagen der Emotionsgeschichte », Revue de l'IFHA [En ligne], Date de recension, mis en ligne le 01 janvier 2016, consulté le 22 septembre 2020 URL : http://journals.openedition.org/ifha/8358 ; DOI : https://doi.org/10.4000/ifha.8358

Ce document a été généré automatiquement le 22 septembre 2020.

(CIFHA 


\title{
Jan Plamper, Geschichte und Gefühl. Grundlagen der Emotionsgeschichte
}

München : Siedler, 2012, 478 p., 29,99€.

\author{
Falk Bretschneider
}

En prenant connaissance du titre de l'ouvrage, le lecteur s'attendra peut-être à lire un manuel l'introduisant à un domaine qui compte actuellement parmi les plus novateurs de l'historiographie allemande: l'histoire des émotions. Il n'en est pourtant rien. Professeur au Goldsmiths College de l'université de Londres, historien de l'Europe de l'Est (il a notamment publié en 2012 une analyse remarquée du culte déployé autour de Staline) et ancien Fellow à l'institut Max-Planck de développement humain à Berlin (autour duquel gravitent actuellement outre-Rhin les recherches en histoire du sensible), l'auteur propose au contraire une réflexion qui est d'abord théorique et méthodologique et qui, comme l'indique le sous-titre de l'ouvrage, cherche à établir les fondements des recherches sur la vie affective. C'est donc davantage une histoire des sciences et des savoirs sur les émotions qu'une introduction à une sous-discipline historiographique bien délimitée (ce que l'histoire des émotions n'est de toute façon pas, ou pas encore, en Allemagne) que J. Plamper entreprend, en se fondant sur - et en s'attaquant à - la structuration binaire d'un champ dont les différentes approches semblent depuis bien longtemps figées dans une opposition ardente entre deux visions, l'une social-constructiviste et l'autre essentialiste, procédant toutes deux d'une question apparemment simple mais complexe et controversée : les émotions sont-elles naturelles ou culturelles, innées ou construites socialement? Sont-elles restées toujours les mêmes à travers les âges (en ne modifiant que leurs modes d'expression) ou bien chaque émotion a-t-elle sa propre histoire qui s'inscrit dans la pluralité historique et culturelle des sociétés?

L'objectif de J. Plamper est de montrer que cette configuration binaire, née avec l'opposition établie par la théorie des sciences autour de 1900 entre sciences de la nature nomothétiques et sciences humaines idiographiques, est une impasse infructueuse. Sa stratégie argumentative consiste à placer l'histoire dans une position intermédiaire - et médiatrice - entre les deux points de vue extrêmes qu'il identifie 
dans les fondements épistémologiques défendus par deux champs disciplinaires, à savoir d'une part l'ethnologie (dominée depuis les années 1970 par un constructivisme radical qui défend la multiplicité des formes et des expressions affectives en fonction de leur contexte historique et culturel) et d'autre part ce que l'on appelle de manière peu précise les «sciences du vivant» (psychologie, médecine, neurosciences, etc.), fondées sur une vision universaliste concevant les émotions essentiellement comme des réactions physiques qu'il est possible de localiser et de visualiser dans des parties précises du cerveau et qui, à en croire les résultats de ces travaux, résultent d'expériences faites par nos ancêtres aux temps archaïques et transmises de génération en génération jusqu'à nos jours.

J. Plamper montre de manière plutôt lucide, dans un style la plupart du temps agréable à lire et en faisant partager une connaissance bibliographique impressionnante, l'évolution de ces deux courants, soumettant ainsi au lecteur une sorte d'état des lieux et une synthèse précise des connaissances acquises dans un domaine dont les historiens n'ont pas forcement été les principaux acteurs. Ce faisant, il ne présente pas seulement une histoire des sciences des émotions, mais il montre également les limites d'une interdisciplinarité rendue impossible par les convictions épistémologiques contradictoires, voire inconciliables, de ceux qui auraient dû la pratiquer. L'objectif du livre, pourtant, va plus loin. Il ne se contente pas d'exposer les résultats d'un double aveuglement disciplinaire, mais souhaite le surmonter en proposant une vision syncrétique s'inspirant de travaux récents (par exemple des deux historiens américains William Reddy et Daniel Lord Smail ou de l'ethnologue Monique Scheer) qui essaient d'élaborer des conceptions unifiantes, soulignant à la fois les fondements naturels de nos réactions affectives et leur inscription dans des contextes historiques et sociaux un état d'esprit qui sous certains aspects rejoint celui des grands débats entre sociologues et psychiatres dans les années 1920 et 1930 quand (par exemple dans le domaine de la criminologie, hantée par l'idée du "criminel né ») les mêmes questions furent déjà posées, et parfois les mêmes réponses données...

Outre le fait que les contours du débat paraissent, en raison de leurs origines plus lointaines, en partie bien connus de certains historiens (ce qui relativise le caractère «innovateur" et "pacificateur» des propositions de J. Plamper), le livre pose cependant un autre problème, notamment pour un public français. Si, dans le bilan historiographique au début de la présentation, Lucien Febvre est cité comme l'un des premiers à s'être intéressés aux émotions collectives et individuelles des acteurs historiques, la recherche française est quasiment occultée partout ailleurs. Certes, à l'instar d'autres historiographies, la version plus particulièrement française d'une "histoire des sensibilités" (relancée dans les années 1950 par Robert Mandrou) a d'abord eu du mal à trouver sa place, mais elle aurait certainement mérité d'être traitée avec davantage d'attention (seul auteur français à être évoqué plus amplement, Alain Corbin est cité p. 83/84 avec son étude sur l'affaire de Hautefaye; en revanche, ni Mandrou ni des médiévistes comme Jean-Claude Schmitt n'apparaissent nulle part alors que l'auteur souligne lui-même à plusieurs reprises l'importance primordiale de l'histoire médiévale pour le développement de l'historiographie sur les émotions, par exemple p. 78) Le lecteur cherche même en vain une référence aux travaux de Stéphane Audoin-Rouzeau, alors que J. Plamper prépare une étude portant sur l'histoire de la peur à l'exemple de l'expérience des soldats russes pendant la Grande Guerre.

De manière involontaire, son livre est donc aussi un signe de la situation paradoxale et un peu préoccupante qui caractérise actuellement les échanges scientifiques entre la 
France et l'Allemagne. Jamais, les conditions (institutionnelles et financières notamment) n'ont été meilleures pour permettre aux historiens des deux côtés du Rhin de se rencontrer et de profiter mutuellement de leurs travaux; et en même temps la perception et la réception mutuelles ne cessent de se restreindre, au profit d'un regard toujours davantage tourné vers le monde anglo-saxon, notamment dans les domaines dont les deux historiographies ressentent elles-mêmes qu'ils sont les plus novatrices, à l'image de l'histoire des émotions.

Vous trouverez la table des matières ici : http://d-nb.info/1021116033/04.

INDEX

Index chronologique : Époque contemporaine

Mots-clés : Histoire des sciences

\section{AUTEUR}

FALK BRETSCHNEIDER

EHESS Paris 